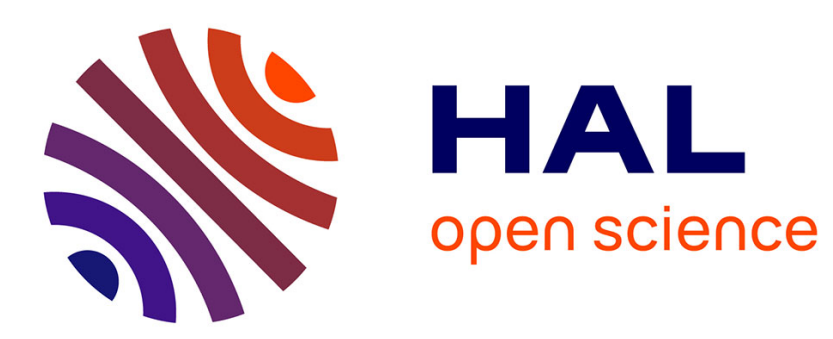

\title{
The magnetic Laplacian in shrinking tubular neighbourhoods of hypersurfaces
}

David Krejcirik, Nicolas Raymond, Matej Tusek

\section{To cite this version:}

David Krejcirik, Nicolas Raymond, Matej Tusek. The magnetic Laplacian in shrinking tubular neighbourhoods of hypersurfaces. Journal of Geometric Analysis, 2015, 25 (4), pp.2546-2564. 10.1007/s12220-014-9525-y . hal-00802483

\section{HAL Id: hal-00802483 https://hal.science/hal-00802483}

Submitted on 19 Mar 2013

HAL is a multi-disciplinary open access archive for the deposit and dissemination of scientific research documents, whether they are published or not. The documents may come from teaching and research institutions in France or abroad, or from public or private research centers.
L'archive ouverte pluridisciplinaire HAL, est destinée au dépôt et à la diffusion de documents scientifiques de niveau recherche, publiés ou non, émanant des établissements d'enseignement et de recherche français ou étrangers, des laboratoires publics ou privés. 


\title{
The magnetic Laplacian in shrinking tubular neighbourhoods of hypersurfaces
}

D. Krejčiř́k ${ }^{a}$ N. Raymond ${ }^{b}$ and M. Tušek ${ }^{c}$

a) Department of Theoretical Physics, Nuclear Physics Institute ASCR, 25068 Rež, Czech Republic; krejcirik@ujf.cas.cz

b) Institut de Recherche Mathématique de Rennes, Université de Rennes 1, 35042 Rennes Cedex, France; nicolas.raymond@univ-rennes1.fr

c) Department of Mathematics, Faculty of Nuclear Sciences and Physical Engineering, Czech Technical University in Prague, Trojanova 13, 12000 Prague 2, Czech Republic; tusekmat@fjfi.cvut.cz

18 March 2013

\begin{abstract}
The Dirichlet Laplacian between two parallel hypersurfaces in Euclidean spaces of any dimension in the presence of a magnetic field is considered in the limit when the distance between the hypersurfaces tends to zero. We show that the Laplacian converges in a norm-resolvent sense to a Schrödinger operator on the limiting hypersurface whose electromagnetic potential is expressed in terms of principal curvatures and the projection of the ambient vector potential to the hypersurface. As an application, we obtain an effective approximation of bound-state energies and eigenfunctions in thin quantum layers.
\end{abstract}




\section{Introduction}

Given a hypersurface $\Sigma$ in the Euclidean space $\mathbb{R}^{d}$ with $d \geq 2$, consider a charged quantum particle constrained to a tubular neighbourhood

$$
\Omega_{\varepsilon}:=\left\{x+t n \in \mathbb{R}^{d} \mid(x, t) \in \Sigma \times(-\varepsilon, \varepsilon)\right\},
$$

where $n$ denotes a unit normal vector field of $\Sigma$. This paper is inspired by the following questions: What is the effective dynamics on the hypersurface approximating the constrained motion when $\varepsilon \rightarrow 0$ ? How does the former depend on the geometry of $\Sigma$ ? To what extent can one recover the geometry of $\Sigma$ from the energy spectrum? What is the role of dimension $d$ ? What is the effect of interaction with an ambient magnetic field $B=* \mathrm{~d} A$ ?

In this paper we model the constrained quantum Hamiltonian by the magnetic Laplacian

$$
(-i \nabla+A)^{2} \quad \text { on } \quad L^{2}\left(\Omega_{\varepsilon}\right)
$$

subject to Dirichlet boundary conditions on $\partial \Omega_{\varepsilon}$, and tackle the questions by developing a singular perturbation theory for a self-adjoint realization of the operator that we concisely denote by $-\Delta_{D, A}^{\Omega_{\varepsilon}}$. Our main result says that

$$
-\Delta_{D, A}^{\Omega_{\varepsilon}}-\left(\frac{\pi}{2 \varepsilon}\right)^{2} \underset{\varepsilon \rightarrow 0}{\longrightarrow} h_{\mathrm{eff}}:=-\Delta_{D, A_{\mathrm{eff}}}^{\Sigma}+V_{\mathrm{eff}}
$$

in a norm-resolvent sense under some additional assumptions on $\Sigma$ and $A$ (see Theorem 7.1 for the precise formulation). Here the kinetic part of the effective Hamiltonian $h_{\text {eff }}$ is the magnetic Laplace-Beltrami operator on $\Sigma$, subject to Dirichlet boundary conditions on $\partial \Sigma$ if the hypersurface is not complete, with $A_{\text {eff }}$ being just the projection of $A$ on $\Sigma$. The geometric potential $V_{\text {eff }}$ depends explicitly on principal curvatures of $\Sigma$, cf (19). The subtraction of the diverging coefficient in (3) is needed in order to "filter out" the transverse oscillations due to the approaching Dirichlet boundaries. It follows that the limiting operator $h_{\text {eff }}$ and its spectrum contain information about both the intrinsic and extrinsic geometry of the hypersurface $\Sigma$. The role of $A_{\text {eff }}$ is best visualized for $d=3$ where $B_{\text {eff }}:=\operatorname{curl} A_{\text {eff }}=n \cdot B$, i.e. only the projection of the ambient magnetic field to the normal bundle of $\Sigma$ plays a role in the limit.

Except for the inclusion of magnetic field - which is a primary motivation for us to write this paper - the aforementioned questions have been considered by several authors during the last two decades, in various settings and with different methods. Indeed, we dare to say that the schematic result (3) for $A=0$, especially the presence of the geometric potential $V_{\text {eff }}$ in the limit, belongs now to an almost common knowledge among spectral geometers and mathematical physicists. Within the range of numerous papers on the subject, let us point out just a few contributions, closest to the present setting, [13, 2, $3,33,4,26,11,10,36,34,35,18,16]$.

Despite of the extensive literature, there does not seem to be any work dealing with (3) in the complete setting of the present paper. More specifically, we are not aware of any work establishing (3) through the norm-resolvent convergence, for arbitrary hypersurfaces (bounded or unbounded), in any dimension and notably with the presence of magnetic field. Furthermore, in this paper we propose a remarkably simple method how to establish the norm-resolvent convergence as a consequence of certain operator inequalities, which we believe 
is of independent interest. Finally, our technique provides an explicit bound on the decay rate of the limit (3).

For $d=2,3$ the result (3) has applications in mesoscopic physics [24, 12]. It can be used to approximate two- or three-dimensional quantum dynamics in long thin nanostructures by the one- or two-dimensional effective Hamiltonian, respectively. Probably the most spectacular phenomenon here is the existence of curvature-induced bound states in unbounded quantum waveguides $(d=2)[9,4$, $15]$ and quantum layers $(d=3)[5,1,22,21,23,25]$. The purely quantum effect can be well understood from (3): since the geometric potential $V_{\text {eff }}$ is always non-positive for $d=2,3, c f(19)$, it represents an attractive interaction and therefore generates discrete eigenvalues below the essential spectrum of $-\Delta_{D, 0}^{\Omega_{\varepsilon}}$.

Our new result involving the magnetic field gives a recipe how to possibly eliminate the disturbing bound states: just embed the device in an ambient magnetic field and employ the repulsive (diamagnetic) feature of the latter [19]. For $d=2$ and $\varepsilon$ fixed, the repulsive nature of the magnetic field in the waveguide context was previously studied in [7]. In higher dimensions $(d \geq 4)$, no robust results about the existence of discrete eigenvalues can be expected, which can be again seen from (3): in addition to different threshold properties of $-\Delta_{D, 0}^{\Sigma}$, $V_{\text {eff }}$ may be repulsive.

The paper is organized as follows. In the forthcoming Section 2 we recall elements of geometry of hypersurfaces and introduce a natural parametrization of $\Omega_{\varepsilon}$. Basic information about the magnetic field in arbitrary dimension and curvilinear coordinates are summarized in Section 3. In Section 4 we introduce the magnetic Laplacian $-\Delta_{D, A}^{\Omega_{\varepsilon}}$ and a unitarily equivalent operator $H$ on an $\varepsilon$-independent Hilbert space, in terms of which the convergence result (3) will be stated. Section 5 is devoted to various two-sided estimates of $H$; here the geometric potential $V_{\text {eff }}$ and the effective Hamiltonian $h_{\text {eff }}$ are encountered for the first time in the present analysis. The main idea how to deduce the normresolvent convergence from the estimates is contained in Section 6, where we also establish the main result of this paper (Theorem 6.3). An alternative version of the result (Theorem 7.1), which is more closer in its spirit to the schematic limit (3), is proved in Section 7 by means of an orthogonal decomposition of the Hilbert space. In Subsection 7.1 we make the general results more explicit for the physically interesting situations of $d=2,3$. Finally, in Section 8 we show how the theorems can be used to deduce a convergence of eigenvalues and eigenfunctions (Corollary 8.1).

\section{Geometric preliminaries}

Let $\Sigma$ be a connected orientable $C^{3}$ hypersurface (compact or non-compact) in $\mathbb{R}^{d}$, with $d \geq 2$, equipped with the Riemannian metric $g$ induced by the embedding. The orientation is specified by a globally defined unit normal vector field $n: \Sigma \rightarrow S^{d-1}$. Without loss of generality, we assume that $\Sigma$ has the same orientation as the ambient Euclidean space $\mathbb{R}^{d}$.

For any $x \in \Sigma$, we introduce the Weingarten map

$$
L: T_{x} \Sigma \rightarrow T_{x} \Sigma:\{\xi \mapsto-\mathrm{d} n(\xi)\} .
$$

Denoting (a bit confusingly) by $x^{1}, \ldots, x^{d-1}$ a local coordinate system of $\Sigma$, $L$ can be identified with a $(1,1)$ mixed tensor having the matrix representa- 
tion $L^{\mu}{ }_{\nu}$ with respect to the coordinate basis $\left(\partial_{x^{1}}, \ldots, \partial_{x^{d-1}}\right)$. Here and in the sequel, we abbreviate $\partial_{x^{\mu}}:=\partial / \partial x^{\mu}$ and assume the range of Greek indices being $1, \ldots, d-1$. The relationship of $L$ with the second fundamental form $h$ of $\Sigma$ is through the formula $L^{\mu}{ }_{\nu}=g^{\mu \rho} h_{\rho \nu}$, where, as usual, $g^{\mu \nu}$ denote the entries of the inverse matrix $\left(g_{\mu \nu}\right)^{-1}$ and the Einstein summation convention is employed. For more details on the geometry of hypersurfaces, we refer for instance to [30, Chap. 1].

The eigenvalues of $L$ are called principal curvatures $\kappa_{1} \ldots \kappa_{d-1}$ of $\Sigma$. It will be convenient to introduce the quantity

$$
\varrho_{m}:=\left(\max \left\{\left\|\kappa_{1}\right\|_{\infty}, \ldots,\left\|\kappa_{d-1}\right\|_{\infty}\right\}\right)^{-1},
$$

with the convention that $\varrho_{m}=\infty$ if all $\kappa_{\mu}$ are identically equal to zero, and $\varrho_{m}=0$ if one of them is unbounded. With help of the principal curvatures, we can construct $d-1$ invariants of $L$ :

$$
K_{\mu}:=\left(\begin{array}{c}
d-1 \\
\mu
\end{array}\right)^{-1} \sum_{\alpha_{1}<\ldots<\alpha_{\mu}} \kappa_{\alpha_{1}} \ldots \kappa_{\alpha_{\mu}}
$$

called $\mu$-th mean curvatures [17].

Given $I:=(-1,1)$ and $\varepsilon>0$, we define a layer $\Omega_{\varepsilon}$ of width $2 \varepsilon$ along $\Sigma$ as the image of the mapping

$$
\mathscr{L}: \Sigma \times I \rightarrow \mathbb{R}^{d}:\{(x, u) \mapsto x+\varepsilon u n\},
$$

i.e., $\Omega_{\varepsilon}:=\mathscr{L}(\Sigma \times I)$. We always assume that $\Omega_{\varepsilon}$ does not overlap itself, i.e., more precisely,

$$
\varepsilon<\varrho_{m} \quad \text { and } \quad \mathscr{L} \quad \text { is injective. }
$$

Since $\varepsilon$ is a small parameter in our setting, the former will be always satisfied provided that the principal curvatures are bounded, while the latter contains a non-trivial hypothesis about the global geometry of $\Sigma$.

The relevance of our basic hypothesis (5) can be seen as follows. The metric $G$ induced by (4) has a block form

$$
G=g \circ(I d-\varepsilon u L)^{2}+\varepsilon^{2} \mathrm{~d} u^{2},
$$

where $I d$ denotes the identity map on $T_{x} \Sigma$. In particular,

$$
\begin{aligned}
|G|:=\operatorname{det}(G) & =\varepsilon^{2}|g|[\operatorname{det}(1-\varepsilon u L)]^{2}=\varepsilon^{2}|g| \prod_{\mu=1}^{d-1}\left(1-\varepsilon u \kappa_{\mu}\right)^{2} \\
& =\varepsilon^{2}|g|\left[1+\sum_{\mu=1}^{d-1}(-\varepsilon u)^{\mu}\left(\begin{array}{c}
d-1 \\
\mu
\end{array}\right) K_{\mu}\right]^{2},
\end{aligned}
$$

with $|g|:=\operatorname{det}(g)$. The formula implies that $G$ is non-singular under the first condition in (5). Consequently, by the inverse function theorem, $\mathscr{L}: \Sigma \times I \rightarrow$ $\Omega_{\varepsilon}$ is a local diffeomorphism, excluding thus "local self-intersections". It will turn into a global diffeomorphism under the additional injectivity hypothesis. It then follows that, under the hypothesis (5), $\Omega_{\varepsilon}$ has indeed the geometrical meaning of the set of points in $\mathbb{R}^{d}$ squeezed between two parallel hypersurfaces at the distance $\varepsilon$ from $\Sigma, c f(1)$. Furthermore, $\Omega_{\varepsilon}$ can be identified with the Riemannian manifold $(\Sigma \times I, G)$. 
Remark 2.1 (On the injectivity assumption). It is possible to consider $(\Sigma \times$ $I, G)$ as an abstract Riemannian manifold where only the hypersurface $\Sigma$ is embedded (or even just immersed) in $\mathbb{R}^{d}$. Then we do not need to assume the second part of hypothesis (5).

Writing $x^{d}:=u$ and $\partial_{x^{d}}:=\partial / \partial u$ with $u \in I,\left(\partial_{x^{1}}, \ldots, \partial_{x^{d}}\right)$ represents a natural coordinate frame for $\Omega_{\varepsilon}$. We thus extend our index convention by assuming the range of Latin indices being $1, \ldots, d$. Then the metric $G$ can be written in the following matrix representation

$$
\left(G_{i j}\right)=\left(\begin{array}{cc}
\left(G_{\mu \nu}\right) & 0 \\
0 & \varepsilon^{2}
\end{array}\right), \quad G_{\mu \nu}=g_{\mu \rho}\left(\delta_{\sigma}^{\rho}-\varepsilon u L_{\sigma}^{\rho}\right)\left(\delta_{\nu}^{\sigma}-\varepsilon u L_{\nu}^{\sigma}\right)
$$

We also introduce $\left(G^{i j}\right):=\left(G_{i j}\right)^{-1}$ and the volume element

$$
\mathrm{d} \Omega_{\varepsilon}:=|G|^{1 / 2} \mathrm{~d} \Sigma \wedge \mathrm{d} u,
$$

where $\mathrm{d} \Sigma:=|g|^{1 / 2} \mathrm{~d} x^{1} \wedge \cdots \wedge \mathrm{d} x^{d-1}$. The reader is warned that, in order not to additionally burden the index notation, we do not make the dependence of $G$ on $\varepsilon$ explicit in this letter, and similarly for several other quantities appearing in the text. We have

$$
C_{-}\left(g_{\mu \nu}\right) \leq\left(G_{\mu \nu}\right) \leq C_{+}\left(g_{\mu \nu}\right) \quad \text { with } \quad C_{ \pm}:=\left(1 \pm \varepsilon \varrho_{m}^{-1}\right)^{2}=1+\mathcal{O}(\varepsilon)
$$

as $\varepsilon \rightarrow 0$.

\section{Magnetic field in curvilinear coordinates}

The identification of $\Omega_{\varepsilon}$ with $(\Sigma \times I, G)$ can be understood as expressing the former in suitable (local) curvilinear coordinates, namely $x \equiv\left(x^{1}, \ldots, x^{d}\right)$. The aim of this section is to introduce a covariant framework for dealing with the magnetic field in these coordinates.

In any dimension, we introduce the "magnetic field" through its vector potential. Let $A \equiv\left(A_{1}, \ldots, A_{d}\right): \mathbb{R}^{d} \rightarrow \mathbb{R}^{d}$ be a $C^{1}$-smooth function and let us call it a vector potential expressed in Cartesian coordinates $y \equiv\left(y^{1}, \ldots, y^{d}\right)$ of $\mathbb{R}^{d}$. It gives rise to a 1 -form

$$
\alpha=A_{i} \mathrm{~d} y^{i} .
$$

Passing locally to other coordinates

$$
y=\Phi(x)
$$

then using the pull-back, the form transforms as follows

$$
\Phi^{*} \alpha=\tilde{A}_{i} \mathrm{~d} x^{i} \quad \text { with } \quad \tilde{A}:=(D \Phi)^{T} A \circ \Phi,
$$

just because $\mathrm{d} y^{i}=\frac{\partial y^{i}}{\partial x^{j}} \mathrm{~d} x^{j}$. This is the way how we express $A$ in local coordinates of $(\Sigma \times I, G)$, using particular charts of $\Sigma$.

The magnetic field is then introduced as the antisymmetric 2-form

$$
\beta:=\mathrm{d} \alpha .
$$

Remark that we have some freedom in the choice of $\alpha$ to get the same $\beta$, which is the well known choice of gauge. Indeed, if $\phi$ stands for a differentiable scalar function, then $\mathrm{d}(\alpha+\mathrm{d} \phi)=\mathrm{d} \alpha=\beta$. In the Cartesian coordinates $y$, we have $\beta_{i j}=\partial_{y^{i}} A_{j}-\partial_{y^{j}} A_{i}$, and similarly for any (curvilinear) coordinates $x$. 


\subsection{Physical realizations}

Now we explain how the general framework is related to the physical notion of magnetic field in low-dimensional Euclidean spaces.

\subsubsection{Case of $d=3$}

The physical object is the magnetic field (magnetic induction) which we identify with the 1 -form $B=B_{i} \mathrm{~d} y^{i}=\tilde{B}_{i} \mathrm{~d} x^{i}$. It obeys the second Maxwell equation (Gauss' law for magnetism) that reads

$$
* \mathrm{~d} * B=0, \quad \text { i.e. } \quad \mathrm{d} * B=0,
$$

where $*$ stands for the Hodge star operator. Recall that on a three-dimensional Riemannian manifold with a metric tensor $G$, we have

$$
* \mathrm{~d} x^{i}=\frac{1}{2}|G|^{1 / 2} G^{i l} \delta_{l k m}^{123} \mathrm{~d} x^{k} \wedge \mathrm{d} x^{m}, \quad *\left(\mathrm{~d} x^{i} \wedge \mathrm{d} x^{j}\right)=|G|^{1 / 2} G^{i l} G^{j k} \delta_{l k m}^{123} \mathrm{~d} x^{m},
$$

where $\delta_{l k m}^{123}$ is the generalized Kronecker symbol [27]. For manifolds equipped with the flat metric, $\mathrm{d}, * \mathrm{~d} *$, and $* \mathrm{~d}$ correspond to the usual grad, div, and curl operators, respectively.

We construct from $B$ a 2 -form $\beta:=* B$. It follows from (11) that $\beta$ is closed, i.e. $\mathrm{d} \beta=0$. Since the ambient space is a Euclidean space, it follows from the Poincaré lemma [31, Chap. 7] that $\beta$ is in fact exact. That is, (10) holds with some 1 -form $\alpha$. Given a 2 -form (10), the magnetic field $B$ can be reconstructed by the formula $B=* \beta=* \mathrm{~d} \alpha=\operatorname{curl} A$, employing the fact that $* *$ is an identity in the present case.

In the Cartesian coordinates, we have

$$
\beta=\beta_{23} \mathrm{~d} y^{2} \wedge \mathrm{d} y^{3}+\beta_{31} \mathrm{~d} y^{3} \wedge \mathrm{d} y^{1}+\beta_{12} \mathrm{~d} y^{1} \wedge \mathrm{d} y^{2} .
$$

The triple (not a vector!)

$$
\gamma:=\left(\beta_{23}, \beta_{31}, \beta_{12}\right)
$$

transforms under the change of coordinates (9) as follows

$$
\tilde{\gamma}:=\left(\tilde{\beta}_{23}, \tilde{\beta}_{31}, \tilde{\beta}_{12}\right)=\operatorname{det}(D \Phi)(D \Phi)^{-1} \gamma .
$$

\subsubsection{Case of $d=2$}

In the planar case, we have $\mathrm{d} \alpha=\tilde{\beta}_{12} \mathrm{~d} x^{1} \wedge \mathrm{d} x^{2}$ and

$$
\tilde{B}:=* \mathrm{~d} \alpha=\tilde{\beta}_{12}|G|^{1 / 2} G^{1 j} G^{2 k} \delta_{j k}^{12}=\tilde{\beta}_{12}|G|^{-1 / 2},
$$

gives the magnitude of the magnetic field in the direction perpendicular to the plane.

\section{The magnetic Laplacian}

Assume $A \in C^{1}\left(\mathbb{R}^{d} ; \mathbb{R}^{d}\right)$. Recall that $\Omega_{\varepsilon}$ is an open subset of $\mathbb{R}^{d}$ as a consequence of (5). The quadratic form

$$
Q[\psi]:=\|(-i \nabla+A) \psi\|_{L^{2}\left(\Omega_{\varepsilon}\right)}^{2}
$$


initially defined on $C_{0}^{\infty}\left(\Omega_{\varepsilon}\right)$ is closable; let us denote by the same symbol $Q$ its closure. For bounded $A$, it is easy to see that $\operatorname{Dom}(Q)=H_{0}^{1}\left(\Omega_{\varepsilon}\right)$. In general, however, we only have $\psi \in \operatorname{Dom}(Q) \Rightarrow|\psi| \in H_{0}^{1}\left(\Omega_{\varepsilon}\right)$, which is a consequence of the diamagnetic inequality [20, Thm. 7.21]. We define the magnetic Laplacian $-\Delta_{D, A}^{\Omega_{\varepsilon}}$ as the unique self-adjoint operator associated with the closure $Q$ via the first representation theorem [14, Thm. VI.2.6].

Remark 4.1 (On the regularity of $A$ ). For the definition of $-\Delta_{D, A}^{\Omega_{\varepsilon}}$ via the quadratic form (15), it is enough to assume $A \in L_{\text {loc }}^{2}\left(\mathbb{R}^{d} ; \mathbb{R}^{d}\right)$. Our smoothness hypothesis will be needed later, $c f(21)$.

As explained in Section 3, let us denote by $\tilde{A}$ the components of the vector potential expressed in the curvilinear coordinates induced by the embedding (4). Moreover, assume

$$
\tilde{A}_{d}=0 .
$$

This may be always achieved by using an appropriate gauge transform, namely,

$$
\tilde{A} \mapsto \tilde{A}-\nabla \int_{0}^{u} \tilde{A}_{d}\left(x^{\mu}, t\right) \mathrm{d} t .
$$

Note that after this gauge transform, $\tilde{A}$ is continuous and it has a continuous derivative in the variable $u$.

Employing the diffeomorphism $\mathscr{L}: \Sigma \times I \rightarrow \Omega_{\varepsilon}$, we may thus identify $-\Delta_{D, A}^{\Omega_{\varepsilon}}$ with an operator $\hat{H}$ on $L^{2}\left(\Sigma \times I, \mathrm{~d} \Omega_{\varepsilon}\right)$ that acts, in the form sense, as

$$
\hat{H}=|G|^{-1 / 2}\left(-i \partial_{x^{\mu}}+\tilde{A}_{\mu}\right)|G|^{1 / 2} G^{\mu \nu}\left(-i \partial_{x^{\nu}}+\tilde{A}_{\nu}\right)-\varepsilon^{-2}|G|^{-1 / 2} \partial_{u}|G|^{1 / 2} \partial_{u} .
$$

More precisely, $\hat{H}$ is the operator associated on $L^{2}\left(\Sigma \times I, \mathrm{~d} \Omega_{\varepsilon}\right)$ with the quadratic form $\hat{h}[\psi]:=Q\left[\hat{U}^{-1} \psi\right], \operatorname{Dom}(\hat{h}):=\hat{U}[\operatorname{Dom}(Q)]$, where $\hat{U} \psi:=\psi \circ \mathscr{L}$.

For our purposes, it will be more convenient to work with a unitarily equivalent operator on a Hilbert space independent of $\varepsilon$. Let us define

$$
J:=\frac{1}{4} \ln \frac{|G|}{|g|}=\frac{1}{2} \sum_{\mu=1}^{d-1} \ln \left(1-\varepsilon u \kappa_{\mu}\right)=\frac{1}{2} \ln \left[1+\sum_{\mu=1}^{d-1}(-\varepsilon u)^{\mu}\left(\begin{array}{c}
d-1 \\
\mu
\end{array}\right) K_{\mu}\right] .
$$

Using the unitary transform

$$
U: L^{2}\left(\Sigma \times I, \mathrm{~d} \Omega_{\varepsilon}\right) \rightarrow L^{2}(\Sigma \times I, \mathrm{~d} \Sigma \wedge \mathrm{d} u):\left\{\psi \mapsto \mathrm{e}^{J} \psi\right\},
$$

we arrive at the unitarily equivalent operator

$$
H:=U \hat{H} U^{-1}=|g|^{-1 / 2}\left(-i \partial_{x^{\mu}}+\tilde{A}_{\mu}\right)|g|^{1 / 2} G^{\mu \nu}\left(-i \partial_{x^{\nu}}+\tilde{A}_{\nu}\right)-\varepsilon^{-2} \partial_{u}^{2}+V,
$$

where

$$
V:=|g|^{-1 / 2} \partial_{x^{i}}\left(|g|^{1 / 2} G^{i j}\left(\partial_{x^{j}} J\right)\right)+\left(\partial_{x^{i}} J\right) G^{i j}\left(\partial_{x^{j}} J\right) .
$$

Again, the expressions should be understood in the sense of forms. $H$ is the operator associated on $L^{2}(\Sigma \times I, \mathrm{~d} \Sigma \wedge \mathrm{d} u)$ with the quadratic form $h[\psi]:=$ $\hat{h}\left[U^{-1} \psi\right], \operatorname{Dom}(h):=U[\operatorname{Dom}(\hat{h})]$. Summing up,

$$
H=U \hat{U}\left(-\Delta_{D, A}^{\Omega_{\varepsilon}}\right) \hat{U}^{-1} U^{-1} .
$$


Remark 4.2 (On the regularity of $\Sigma$ ). Note that while $\hat{H}$ can be introduced under the conventional $C^{2}$ smoothness assumption about $\Sigma$, it is because of the operator $H$ why we actually require $C^{3}$. Indeed, we need to differentiate the principal curvatures of $\Sigma$ appearing in $J$ in order to define $H$, even if this is understood as an operator associated with the quadratic form $h$. To proceed without the additional regularity, one can apply the recent idea of refined $\varepsilon$ dependent smoothing of curvatures [16], but the overall analysis would become much more cumbersome.

Henceforth, we work in the $\varepsilon$-independent Hilbert space $L^{2}(\Sigma \times I, \mathrm{~d} \Sigma \wedge$ $\mathrm{d} u$ ), the norm and inner product of which will be denoted by $\|\cdot\|$ and $\langle\cdot, \cdot\rangle$, respectively. The norm and inner product in $L^{2}(\Sigma, \mathrm{d} \Sigma)$ will be denoted by $\|\cdot\|_{g}$ and $\langle\cdot, \cdot\rangle_{g}$, respectively.

\section{Comparison operators}

Applying (7) to $\left\langle\left(\partial_{x^{\mu}} J\right) \psi-\left(\partial_{x^{\mu}}+i A_{\mu}\right) \psi, G^{\mu \nu}\left[\left(\partial_{x^{\nu}} J\right) \psi-\left(\partial_{x^{\nu}}+i A_{\nu}\right) \psi\right]\right\rangle$, with $\psi \in C_{0}^{1}(\Sigma \times I)$, we obtain crucial bounds

$$
H_{-} \leq H \leq H_{+}
$$

in the form sense, with the comparison operators

$$
H_{ \pm}:=C_{\mp}^{-1}\left(|g|^{-1 / 2}\left(-i \partial_{x^{\mu}}+\tilde{A}_{\mu}\right)|g|^{1 / 2} g^{\mu \nu}\left(-i \partial_{x^{\nu}}+\tilde{A}_{\nu}\right)+v_{1}\right)-\varepsilon^{-2} \partial_{u}^{2}+V_{2}
$$

where

$$
\begin{aligned}
& v_{1}:=\Delta_{g} J+\left|\nabla_{g} J\right|_{g}^{2}=\frac{1}{2} \frac{\Delta_{g} f}{1+f}-\frac{1}{4} \frac{\left|\nabla_{g} f\right|_{g}^{2}}{(1+f)^{2}}, \quad f:=\sum_{\mu=1}^{d-1}(-\varepsilon u)^{\mu}\left(\begin{array}{c}
d-1 \\
\mu
\end{array}\right) K_{\mu}, \\
& V_{2}:=\varepsilon^{-2}\left[\partial_{u}^{2} J+\left(\partial_{u} J\right)^{2}\right]=-\frac{1}{2} \sum_{\mu=1}^{d-1} \frac{\kappa_{\mu}^{2}}{\left(1-\varepsilon u \kappa_{\mu}\right)^{2}}+\frac{1}{4}\left(\sum_{\mu=1}^{d-1} \frac{\kappa_{\mu}}{1-\varepsilon u \kappa_{\mu}}\right)^{2} .
\end{aligned}
$$

Here we have introduced coordinate-free notations

$$
\Delta_{g} f:=|g|^{-1 / 2} \partial_{x^{\mu}}\left(|g|^{1 / 2} g^{\mu \nu} \partial_{x^{\nu}} f\right), \quad\left|\nabla_{g} f\right|_{g}:=\sqrt{\left(\partial_{x^{\mu}} f\right) g^{\mu \nu}\left(\partial_{x^{\nu}} f\right)} .
$$

In order to give a meaning to the Laplacian of curvatures in the definition of the potential $v_{1}$, we need to strengthen our regularity assumptions about $\Sigma$. Henceforth, we assume

$$
\left|\nabla_{g} \kappa_{\mu}\right|_{g}, \Delta_{g} \kappa_{\mu} \in L^{\infty}(\Sigma)
$$

which is equivalent to $\left|\nabla_{g} K_{\mu}\right|_{g}, \Delta_{g} K_{\mu}, \in L^{\infty}(\Sigma)$. Recall that the assumption $\kappa_{\mu} \in L^{\infty}(\Sigma)$ is implicit in the first condition of (5). Under the hypotheses, we have

$$
v_{1}=\mathcal{O}(\varepsilon), \quad V_{2}=V_{\text {eff }}+\mathcal{O}(\varepsilon)
$$

uniformly as $\varepsilon \rightarrow 0$, where

$$
V_{\mathrm{eff}}:=-\frac{1}{2} \sum_{\mu=1}^{d-1} \kappa_{\mu}^{2}+\frac{1}{4}\left(\sum_{\mu=1}^{d-1} \kappa_{\mu}\right)^{2} .
$$


From (17) we deduce cruder bounds

$$
H^{-} \leq H \leq H^{+}
$$

with

$$
H^{ \pm}:=C_{\mp}^{-1}\left(|g|^{-1 / 2}\left(-i \partial_{x^{\mu}}+\tilde{A}_{\mu}\right)|g|^{1 / 2} g^{\mu \nu}\left(-i \partial_{x^{\nu}}+\tilde{A}_{\nu}\right)+V_{\mathrm{eff}}\right)-\varepsilon^{-2} \partial_{u}^{2} \pm C_{0},
$$

where $C_{0}$ is a positive constant such that $C_{0}=\mathcal{O}(\varepsilon)$ as $\varepsilon \rightarrow 0$. Remark that this result was obtained earlier in [5,8] for the special case $d=3$ and $A=0$.

If $\tilde{A}_{\mu}$ is independent of the "transverse" coordinate $u$, then $H^{ \pm}$are decoupled on $L^{2}(\Sigma, \mathrm{d} \Sigma) \otimes L^{2}(I, \mathrm{~d} u)$. Our next aim will be to find decoupled comparison operators in the general case. To this purpose define an $\varepsilon$-independent quantity $\hat{A}(x, t):=\tilde{A}(x, t / \varepsilon)$ and expand it in the last variable into the Taylor series,

$$
\tilde{A}(x, u)=\hat{A}(x, \varepsilon u)=\hat{A}(x, 0)+\varepsilon u \partial_{d} \hat{A}(x, \xi(x, u))=: \tilde{A}(x, 0)+\varepsilon A^{\prime}(x, u),
$$

with some $\xi(x, u) \in(-\varepsilon, \varepsilon)$. It is important to stress that $A^{\prime}$ depends on $\varepsilon$ only through $\xi$, which measures the actual distance from $\Sigma$ in $\mathbb{R}^{d}$.

For any trial function $\psi \in C_{0}^{1}(\Sigma \times I)$, we have

$$
\begin{aligned}
\left\langle\left(-i \partial_{x^{\mu}}+\tilde{A}_{\mu}\right) \psi, g^{\mu \nu}\left(-i \partial_{x^{\nu}}+\tilde{A}_{\nu}\right) \psi\right\rangle_{g} \\
=\left\langle\left(-i \partial_{x^{\mu}}+\tilde{A}_{\mu}(\cdot, 0)\right) \psi, g^{\mu \nu}\left(-i \partial_{x^{\nu}}+\tilde{A}_{\nu}(\cdot, 0)\right) \psi\right\rangle_{g} \\
\quad+2 \varepsilon \Re\left\langle A_{\mu}^{\prime} \psi, g^{\mu \nu}\left(-i \partial_{x^{\nu}}+\tilde{A}_{\nu}(\cdot, 0)\right) \psi\right\rangle_{g}+\varepsilon^{2}\left\langle A_{\mu}^{\prime} \psi, g^{\mu \nu} A_{\nu}^{\prime} \psi\right\rangle_{g} .
\end{aligned}
$$

By the Cauchy-Schwarz and the Young inequalities,

$$
\begin{aligned}
& \left|\left\langle A_{\mu}^{\prime} \psi, g^{\mu \nu}\left(-i \partial_{x^{\nu}}+\tilde{A}_{\nu}(., 0)\right) \psi\right\rangle_{g}\right| \\
\leq & \frac{1}{2}\left\langle A_{\mu}^{\prime} \psi, g^{\mu \nu} A_{\nu}^{\prime} \psi\right\rangle_{g}+\frac{1}{2}\left\langle\left(-i \partial_{x^{\mu}}+\tilde{A}_{\mu}(., 0)\right) \psi, g^{\mu \nu}\left(-i \partial_{x^{\nu}}+\tilde{A}_{\nu}(., 0)\right) \psi\right\rangle_{g} .
\end{aligned}
$$

Consequently,

$$
h^{-} \leq|g|^{-1 / 2}\left(-i \partial_{x^{\mu}}+\tilde{A}_{\mu}\right)|g|^{1 / 2} g^{\mu \nu}\left(-i \partial_{x^{\nu}}+\tilde{A}_{\nu}\right) \leq h^{+}
$$

where

$h^{ \pm}:=(1 \pm \varepsilon)|g|^{-1 / 2}\left(-i \partial_{x^{\mu}}+\tilde{A}_{\mu}(., 0)\right)|g|^{1 / 2} g^{\mu \nu}\left(-i \partial_{x^{\nu}}+\tilde{A}_{\nu}(., 0)\right)+\left( \pm \varepsilon+\varepsilon^{2}\right) A_{\mu}^{\prime} g^{\mu \nu} A_{\nu}^{\prime}$.

Putting this into (20), we arrive at the following result.

Proposition 5.1. In addition to (5) and (18), let us assume

$$
A_{\mu}^{\prime} g^{\mu \nu} A_{\nu}^{\prime} \in L^{\infty}\left(\Sigma \times\left(-\varrho_{m}, \varrho_{m}\right)\right),
$$

where $A_{\mu}^{\prime}$ is introduced in (21). Then

$$
H_{0}^{-} \leq H \leq H_{0}^{+}
$$

with

$$
\begin{aligned}
H_{0}^{ \pm}:= & \mathcal{C}_{ \pm}\left(|g|^{-1 / 2}\left(-i \partial_{x^{\mu}}+\tilde{A}_{\mu}(., 0)\right)|g|^{1 / 2} g^{\mu \nu}\left(-i \partial_{x^{\nu}}+\tilde{A}_{\nu}(., 0)\right)+V_{\text {eff }}\right) \\
& -\varepsilon^{-2} \partial_{u}^{2} \pm \mathcal{C}_{0}, \\
\text { where } \mathcal{C}_{ \pm}:= & (1 \pm \varepsilon) C_{\mp}^{-1}=1+\mathcal{O}(\varepsilon) \text { and } \mathcal{C}_{0}=\mathcal{O}(\varepsilon) \text { as } \varepsilon \rightarrow 0 .
\end{aligned}
$$


Remark 5.2. If $d=3$, then $\tilde{B}_{\mu} \in L^{\infty}(\Sigma \times I)$ implies $A_{\mu}^{\prime} \in L^{\infty}\left(\Sigma \times\left(-\varrho_{m}, \varrho_{m}\right)\right)$.

Proposition 5.1 suggests that for small values of $\varepsilon, H$ behaves like

$$
H_{0}=h_{\mathrm{eff}}-\varepsilon^{-2} \partial_{u}^{2} \simeq h_{\mathrm{eff}} \otimes 1+1 \otimes\left(-\varepsilon^{-2} \partial_{u}^{2}\right)
$$

on $L^{2}(\Sigma \times I, \mathrm{~d} \Sigma \wedge d u) \simeq L^{2}(\Sigma, \mathrm{d} \Sigma) \otimes L^{2}(I, \mathrm{~d} u)$ with the effective Hamiltonian

$$
h_{\mathrm{eff}}:=|g|^{-1 / 2}\left(-i \partial_{x^{\mu}}+\tilde{A}_{\mu}(., 0)\right)|g|^{1 / 2} g^{\mu \nu}\left(-i \partial_{x^{\nu}}+\tilde{A}_{\nu}(., 0)\right)+V_{\mathrm{eff}} .
$$

In particular, applying the minimax principle to (23), one can show that any eigenvalue of $H$ that lies below the essential spectrum is well approximated by an eigenvalue of $H_{0}$ for $\varepsilon$ small enough. To say more about the convergence of the spectrum and the eigenfunctions, we need the norm resolvent convergence of the respective operators, which we establish in the next section.

\section{The norm-resolvent convergence}

As $\varepsilon \rightarrow 0$, the second term of $H_{0}$ diverges and consequently the spectrum of $H_{0}^{ \pm}$ explodes. To renormalize it, let us introduce $E_{m}$ as the $m$-th eigenvalue of $-\partial_{u}^{2}$ on $L^{2}(I)$, subject to Dirichlet boundary condition, i.e. $E_{m}=(m \pi / 2)^{2}$ with $m \in \mathbb{N}$, and subtract $\varepsilon^{-2} E_{1}$ from both $H_{0}^{ \pm}$and $H$ :

$$
H_{\text {ren }}:=H-\varepsilon^{-2} E_{1}, \quad H_{0, \text { ren }}:=H_{0}-\varepsilon^{-2} E_{1} .
$$

With this renormalization, (23) reads

$$
\mathcal{C}_{-} h_{\text {eff }}-\varepsilon^{-2}\left(\partial_{u}^{2}+E_{1}\right)-\mathcal{C}_{0} \leq H_{\text {ren }} \leq \mathcal{C}_{+} h_{\text {eff }}-\varepsilon^{-2}\left(\partial_{u}^{2}+E_{1}\right)+\mathcal{C}_{0} .
$$

Next, choose a constant $k$ large enough that for all $\varepsilon$ smaller than some $\varepsilon_{0}>0$,

$$
h_{\mathrm{eff}}+k \geq 1 \quad \text { and } \quad \mathcal{C}_{-} h_{\mathrm{eff}}-\mathcal{C}_{0}+k \geq 1 .
$$

Such $k$ always exists, since $V_{\text {eff }}$ is bounded. Consequently, $\left\|\left(H_{\text {ren }}+k\right)^{-1}\right\| \leq 1$ and $\left\|\left(H_{0, \text { ren }}+k\right)^{-1}\right\| \leq 1$.

Using [14, Thm. VI.2.21], we deduce from (26) the resolvent bounds

$$
\begin{aligned}
& {\left[\mathcal{C}_{+} h_{\mathrm{eff}}-\varepsilon^{-2}\left(\partial_{u}^{2}+\right.\right.}\left.\left.E_{1}\right)+\mathcal{C}_{0}+k\right]^{-1}-\left(H_{0, \text { ren }}+k\right)^{-1} \\
& \leq\left(H_{\mathrm{ren}}+k\right)^{-1}-\left(H_{0, \text { ren }}+k\right)^{-1} \leq \\
& {\left[\mathcal{C}_{-} h_{\mathrm{eff}}-\varepsilon^{-2}\left(\partial_{u}^{2}+E_{1}\right)-\mathcal{C}_{0}+k\right]^{-1}-\left(H_{0, \text { ren }}+k\right)^{-1} }
\end{aligned}
$$

Our next strategy is to apply the following pair of observations:

Lemma 6.1. Let $T$ be a positive self-adjoint operator and let $S$ stands for a symmetric operator that is relatively form bounded by $T$ with the relative bound $a<1$ and the other constant $b=0$. Then $T^{-1 / 2} S T^{-1 / 2}$ (as a quadratic form) corresponds to a bounded operator L. Moreover $\|L\|<1$ and

$$
(T+S)^{-1}=T^{-1 / 2}(1+L)^{-1} T^{-1 / 2} \text {. }
$$

This immediately implies

$$
\left\|(T \dot{+} S)^{-1}-T^{-1}\right\| \leq \frac{\left\|T^{-1}\right\|\|L\|}{1-\|L\|} .
$$


Lemma 6.2. Let $\left\{L_{n}\right\},\left\{L_{n}^{ \pm}\right\}$be sequences of bounded self-adjoint operators and

$$
L_{n}^{-} \leq L_{n} \leq L_{n}^{+} .
$$

Then $\left\|L_{n}\right\| \leq \max \left\{\left\|L_{n}^{+}\right\|,\left\|L_{n}^{-}\right\|\right\}$. In particular, if $\lim _{n \rightarrow \infty}\left\|L_{n}^{ \pm}\right\|=0$, then also $\lim _{n \rightarrow \infty}\left\|L_{n}\right\|=0$.

Lemma 6.1 is just a special case of [32, Thm. 6.25] (see also [29] for similar manipulations), while Lemma 6.2 is a direct consequence of the minimax principle.

In Lemma 6.1 put

$$
T:=H_{0, \text { ren }}+k, \quad S:=H_{0}^{-}-H_{0}=\left(\mathcal{C}_{-}-1\right) h_{\mathrm{eff}}-\mathcal{C}_{0}
$$

(here we view $h_{\text {eff }}$ as $h_{\text {eff }} \otimes 1$ on $L^{2}(\Sigma, \mathrm{d} \Sigma) \otimes L^{2}(I, \mathrm{~d} u)$ ). Then for all $\psi \in$ $\operatorname{Dom}\left(T^{1 / 2}\right)$, we have

$$
\begin{aligned}
|\langle\psi, S \psi\rangle| & =\left|\left\langle\psi,\left[\left(\mathcal{C}_{-}-1\right)\left(h_{\mathrm{eff}}+k\right)+\left(1-\mathcal{C}_{-}\right) k-\mathcal{C}_{0}\right] \psi\right\rangle\right| \\
& \leq\left|\mathcal{C}_{-}-1\right|\left\langle\psi,\left(h_{\mathrm{eff}}+k\right) \psi\right\rangle+\left|\left(1-\mathcal{C}_{-}\right) k-\mathcal{C}_{0}\right|\langle\psi, \psi\rangle \\
& \leq\left(1-\mathcal{C}_{-}+\left|\left(1-\mathcal{C}_{-}\right) k-\mathcal{C}_{0}\right||| T^{-1} \|\right)\langle\psi, T \psi\rangle .
\end{aligned}
$$

Here $\langle\psi, S \psi\rangle$ is understood as the action on $\psi$ of the quadratic form associated with $S$ and similarly for the other operators. In the second inequality we have used that $h_{\text {eff }} \leq H_{0, \text { ren }}$. Since

$$
a_{-}:=1-\mathcal{C}_{-}+\left|\left(1-\mathcal{C}_{-}\right) k-\mathcal{C}_{0}\right|\left\|\left(H_{0, \text { ren }}+k\right)^{-1}\right\|=\mathcal{O}(\varepsilon) \quad \text { as } \quad \varepsilon \rightarrow 0,
$$

we see that $a_{-}<1$ for $\varepsilon$ small enough, and from (28) we infer that

$$
\left\|\left(\mathcal{C}_{-} h_{\text {eff }}-\varepsilon^{-2}\left(\partial_{u}^{2}+E_{1}\right)-\mathcal{C}_{0}+k\right)^{-1}-\left(H_{0, \text { ren }}+k\right)^{-1}\right\| \leq \frac{\left\|\left(H_{0, \text { ren }}+k\right)^{-1}\right\| a_{-}}{1-a_{-}} .
$$

In a similar manner we obtain that whenever

$$
a_{+}:=\mathcal{C}_{+}-1+\left|\left(1-\mathcal{C}_{+}\right) k+\mathcal{C}_{0}\right|||\left(H_{0, \text { ren }}+k\right)^{-1} \|=\mathcal{O}(\varepsilon)<1,
$$

then

$\left\|\left(\mathcal{C}_{+} h_{\mathrm{eff}}-\varepsilon^{-2}\left(\partial_{u}^{2}+E_{1}\right)+\mathcal{C}_{0}+k\right)^{-1}-\left(H_{0, \text { ren }}+k\right)^{-1}\right\| \leq \frac{\left\|\left(H_{0, \text { ren }}+k\right)^{-1}\right\| a_{+}}{1-a_{+}}$.

Putting these two estimates together with Lemma 6.2 and (27), we arrive at the key result of this paper:

Theorem 6.3. Assume (5), (18) and (22). Then

$$
\begin{aligned}
\left\|\left(H_{\text {ren }}+k\right)^{-1}-\left(H_{0, \text { ren }}+k\right)^{-1}\right\| & \leq\left\|\left(H_{0, \text { ren }}+k\right)^{-1}\right\| \max \left\{\frac{a_{-}}{1-a_{-}}, \frac{a_{+}}{1-a_{+}}\right\} \\
& =\mathcal{O}(\varepsilon) \quad \text { as } \quad \varepsilon \rightarrow 0 .
\end{aligned}
$$

Remark 6.4 (Gauge invariance). Recall that we have worked in a special gauge, namely (16). If we had started with another one that differs by $\nabla \phi$ for some real differentiable function $\phi$, we would have obtain

$$
-\Delta_{D, A+\nabla \phi}^{\Omega_{\varepsilon}}=(-i \nabla+A+\nabla \phi)^{2}=\mathrm{e}^{-i \phi}\left(-\Delta_{D, A}^{\Omega_{\varepsilon}}\right) \mathrm{e}^{i \phi} .
$$


This implies that when passing to this new gauge, $\hat{H}$ and $H$ must be interchanged for $\mathrm{e}^{-i \tilde{\phi}} \hat{H} \mathrm{e}^{i \tilde{\phi}}$ and $\mathrm{e}^{-i \tilde{\phi}} H \mathrm{e}^{i \tilde{\phi}}$, respectively, where $\tilde{\phi}:=\phi \circ \mathscr{L}$. All the weak estimates for the comparison operators above may be sandwiched by $\mathrm{e}^{\mp i \tilde{\phi}}$ and they still remain valid. Consequently, we obtain the inequality of Theorem 6.3 for the pair $\mathrm{e}^{-i \tilde{\phi}} H_{\text {ren }} \mathrm{e}^{i \tilde{\phi}}$ and $\mathrm{e}^{-i \tilde{\phi}} H_{0, \text { ren }} \mathrm{e}^{i \tilde{\phi}}$ with exactly the same upper bound.

Remark 6.5 (Electric field). If we had started with a full Schrödinger operator in $\Omega_{\varepsilon}$, i.e., $-\Delta_{D, A}^{\Omega_{\varepsilon}}+V$, where the scalar potential $V$ that represents an ambient electric field is such that $\partial_{y^{j}} V$ is bounded on $\Omega_{\varrho_{m}}$, we would have arrived at the same convergence result with the effective Hamiltonian (25) merely modified by adding the projection $(V \circ \mathscr{L})(\cdot, 0)$. This can be established quite straightforwardly with the aid of the following Taylor expansion

$$
(V \circ \mathscr{L})(x, u)=(V \circ \mathscr{L})(x, 0)+\varepsilon u n^{j}(x)\left(\partial_{y^{j}} V \circ \mathscr{L}\right)(x, \xi)
$$

that holds for any $x \in \Sigma$, and where $\xi=\xi(x, u) \in I$.

However, if $V$ is singular on $\Sigma$, the situation is much more delicate. For a model example see [6], where a planar layer with the Coulomb potential and without any magnetic field was considered.

\section{A dimensional reduction}

In this section we derive a variant of Theorem 6.3 by replacing $H_{0 \text {,ren }}$ directly by the $(d-1)$-dimensional effective Hamiltonian $h_{\text {eff }}, c f(25)$. It requires certain prerequisites and identifications, because $H_{\text {ren }}$ and $h_{\text {eff }}$ act on different Hilbert spaces.

Let us denote by $\chi_{m}$ the eigenfunction of $-\partial_{u}^{2}$ (subject to Dirichlet boundary conditions) corresponding to $E_{m}$ In particular, we choose

$$
\chi_{1}(u):=\cos (\pi u / 2) .
$$

We decompose our Hilbert space into an orthogonal sum

$$
L^{2}(\Sigma \times I, \mathrm{~d} \Sigma \wedge d u)=\mathcal{H}_{1} \oplus \mathcal{H}_{1}^{\perp},
$$

where the subspace $\mathcal{H}_{1}$ consists of functions of the form

$$
\psi_{1}\left(x^{1}, \ldots, x^{d}\right)=\varphi\left(x^{1}, \ldots, x^{d-1}\right) \chi_{1}\left(x^{d}\right) .
$$

Given any $\psi \in L^{2}(\Sigma \times I, \mathrm{~d} \Sigma \wedge d u)$, we have the decomposition with $\psi_{1} \in \mathcal{H}_{1}$ and $\phi \in \mathcal{H}_{1}^{\perp}$. More specifically, $\psi_{1}=P_{1} \psi$ and $\phi=Q \psi$, where $P_{1}:=\chi_{1}\left\langle\chi_{1}, \cdot\right\rangle_{L^{2}(I)}$ is the projection on the lowest transverse mode and $Q:=1-P_{1}$. The mapping $\iota: \varphi \mapsto \psi_{1}$ is an isomorphism of $L^{2}(\Sigma, d \Sigma)$ onto $\mathcal{H}_{1}$. Hence, with an abuse of notations, we may identify any operator $h$ on $L^{2}(\Sigma, d \Sigma)$ with the operator $\iota h \iota^{-1}$ acting on $\mathcal{H}_{1} \subset L^{2}(\Sigma \times I, \mathrm{~d} \Sigma \wedge d u)$. Having this convention in mind, we can write $P_{1} H_{0, \text { ren }} P_{1}=h_{\text {eff }}$ and have the following decomposition

$$
\begin{aligned}
H_{0, \text { ren }} & =\left(\begin{array}{cc}
h_{\text {eff }} & 0 \\
0 & Q H_{0, \text { ren }} Q
\end{array}\right), \\
\left(H_{0, \text { ren }}+k\right)^{-1} & =\left(\begin{array}{cc}
\left(h_{\mathrm{eff}}+k\right)^{-1} & 0 \\
0 & \left(Q H_{0, \text { ren }} Q+k\right)^{-1}
\end{array}\right),
\end{aligned}
$$


as operators on $\mathcal{H}_{1} \oplus \mathcal{H}_{1}^{\perp}$.

Since

$$
Q\left(H_{0, \mathrm{ren}}+k\right) Q=Q\left(h_{\mathrm{eff}}+k\right) Q-\varepsilon^{-2} Q\left(\partial_{u}^{2}+E_{1}\right) Q \geq \varepsilon^{-2}\left(E_{2}-E_{1}\right)=\frac{3 \pi^{2}}{4 \varepsilon^{2}},
$$

we obtain

$$
\left\|\left(H_{0, \mathrm{ren}}+k\right)^{-1}-\left(h_{\mathrm{eff}}+k\right)^{-1} \oplus 0\right\|=\left\|\left(Q H_{0, \mathrm{ren}} Q+k\right)^{-1}\right\| \leq \frac{4 \epsilon^{2}}{3 \pi^{2}} .
$$

This together with Theorem 6.3 implies

Theorem 7.1. Under the hypotheses of Theorem 6.3,

$$
\left\|\left(H_{\mathrm{ren}}+k\right)^{-1}-\left(h_{\mathrm{eff}}+k\right)^{-1} \oplus 0\right\|=\mathcal{O}(\varepsilon) \quad \text { as } \quad \varepsilon \rightarrow 0 .
$$

\subsection{Physical realizations}

The message of the general result is that the (unitarily transformed and suitably renormalized) magnetic Laplacian $-\Delta_{D, A}^{\Omega_{\varepsilon}}$ in the layer $\Omega_{\varepsilon}$ behaves for small $\varepsilon$ as the effective Hamiltonian $h_{\text {eff }}$ on the underlying hypersurface. The coefficients of $h_{\text {eff }}$ depend on curvatures of $\Sigma$ and on the ambient vector potential projected to $\Sigma$, cf (25). Let us interpret this dependence in physically interesting situations of low-dimensional Euclidean spaces. We refer to Section 3.1 for the notation concerning the magnetic field in our setting.

\subsubsection{Case of $d=3$}

In this case, $V_{\text {eff }}=K_{2}-K_{1}^{2}=-\frac{1}{4}\left(\kappa_{1}-\kappa_{2}\right)^{2}$, where $K_{1}=\frac{1}{2}\left(\kappa_{1}+\kappa_{2}\right)$ and $K_{2}=\kappa_{1} \kappa_{2}$ are the familiar mean and Gauss curvatures of $\Sigma$, respectively. Hence, if $\Sigma$ is not a part of a plane or a sphere, then $V_{\text {eff }}$ always represents an attractive interaction (i.e. $V_{\text {eff }}$ is non-positive and non-trivial). The kinetic part of $h_{\mathrm{eff}}$ is the magnetic Laplace-Beltrami operator on the two-dimensional surface $\Sigma$ with a vector potential associated with the 1 -form

$$
\alpha_{\text {eff }}:=\tilde{A}_{1}(\cdot, 0) \mathrm{d} x^{1}+\tilde{A}_{2}(\cdot, 0) \mathrm{d} x^{2} .
$$

Consequently, $\mathrm{d} \alpha_{\text {eff }}=\tilde{\beta}_{12}(\cdot, 0) \mathrm{d} x^{1} \wedge \mathrm{d} x^{2}$ with $\tilde{\beta}_{12}(\cdot, 0)=\partial_{1} \tilde{A}_{2}(\cdot, 0)-\partial_{2} \tilde{A}_{2}(\cdot, 0)$. Using the transformation rule (13), we get

$$
\left.\tilde{\beta}_{12}\right|_{u=0}=\left.\left.\operatorname{det}(D \mathcal{L})\right|_{u=0}\left(\left.(D \mathcal{L})\right|_{u=0}\right)_{3 j}^{-1} B_{j}\right|_{u=0}=\left.|g|^{1 / 2} n \cdot B\right|_{u=0} \cdot
$$

Here the second equality is a consequence of a tedious computation which can be greatly simplified by using the algebraic formula

$$
M^{-1}=\frac{1}{\operatorname{det} M}\left(\begin{array}{l}
\left(\vec{m}_{2} \times \vec{m}_{3}\right)^{T} \\
\left(\vec{m}_{3} \times \vec{m}_{1}\right)^{T} \\
\left(\vec{m}_{1} \times \vec{m}_{2}\right)^{T}
\end{array}\right) \quad \text { for } \quad M:=\left(\vec{m}_{1}, \vec{m}_{2}, \vec{m}_{3}\right),
$$

where $\vec{m}_{i}$ are three-dimensional column vectors. Finally, using (14) for the twodimensional metric $g$, we conclude that the magnetic field associated with $\alpha_{\text {eff }}$ is given by

$$
B_{\text {eff }}:=* \mathrm{~d} \alpha_{\text {eff }}=\left.n \cdot B\right|_{u=0} .
$$

Therefore in the limit $\varepsilon \rightarrow 0$, a particle confined to the layer $\Omega_{\varepsilon}$ is affected only by the projection of the original magnetic field into the direction that is normal to the underlying surface $\Sigma$. 


\subsubsection{Case of $d=2$}

In this case, $V_{\text {eff }}=-\frac{1}{4} \kappa_{1}^{2}$ is again attractive. Unless $\Sigma$ is a closed curve (i.e. homeomorphic to a circle), there is no magnetic effect in the limit $\varepsilon \rightarrow 0$, because the presence of $\tilde{A}_{1}(\cdot, 0)$ in the one-dimensional kinetic part of $h_{\text {eff }}$ can be always gauged out.

\section{Spectral consequences}

We have obtained the norm-resolvent convergences of $H_{\text {ren }}$ to $H_{0 \text {,ren }}$ or $h_{\text {eff }}$, respectively, for a special value (namely $-k$ ) of the spectral parameter from the resolvent set. However, for sufficiently small $\varepsilon$ (the particular threshold value depends on the chosen value of the spectral parameter), these results may be extended to the resolvent sets of $H_{0, \text { ren }}$ or $h_{\text {eff }}$, respectively, $c f$ [14, Eq. IV.3.10].

Now, let $\mu$ be an isolated eigenvalue of $h_{\text {eff }}$ of finite multiplicity $N$ and denote by $\varphi_{1}, \ldots, \varphi_{N}$ the corresponding (orthogonal) eigenfunctions. Let $\Gamma$ be a contour in the complex plane of radius smaller than the isolation distance of $\mu$ centered at $\mu$. Then for all sufficiently small $\varepsilon$, every point of $\Gamma$ lies also in the resolvent set of $H_{\text {ren }}$. Hence, it makes sense to define the eigenprojections

$$
P:=-\frac{1}{2 \pi i} \int_{\Gamma}\left(H_{\mathrm{ren}}-\xi\right)^{-1} \mathrm{~d} \xi, \quad P_{\mathrm{eff}}:=-\frac{1}{2 \pi i} \int_{\Gamma}\left(h_{\mathrm{eff}}-\xi\right)^{-1} \mathrm{~d} \xi .
$$

Using Theorem 7.1, we obtain that $\left\|P-P_{\text {eff }}\right\|=\mathcal{O}(\varepsilon)$ as $\varepsilon \rightarrow 0$. In particular, $\left\|P-P_{\text {eff }}\right\|<1$ for $\varepsilon$ small enough. We conclude that for these values of $\varepsilon$, the number of eigenvalues of $H_{\text {ren }}$ (counting possible multiplicities) lying inside $\Gamma$ coincides with $N$, cf [14, Thm. I.6.32]. Moreover, $\psi_{n}^{\varepsilon}:=P\left(\varphi_{n} \otimes \chi_{1}\right)$, with $n=1, \ldots, N$, are eigenfunctions of $H_{\text {ren }}$ associated with these eigenvalues of $H_{\text {ren }}$, which we denote by $\lambda_{1}^{\varepsilon}, \ldots \lambda_{N}^{\varepsilon}$ (they are not necessarily sorted in a non-decreasing order, but they are counted according to multiplicities).

Corollary 8.1. Under the hypotheses of Theorem 6.3 and with the above notation, for every $n \in\{1, \ldots, N\}$, we have

$$
\left|\lambda_{n}^{\varepsilon}-\mu\right|=\mathcal{O}(\varepsilon) \quad \text { and } \quad\left\|\psi_{n}^{\varepsilon}-\varphi_{n} \otimes \chi_{1}\right\|=\mathcal{O}(\varepsilon) \quad \text { as } \quad \varepsilon \rightarrow 0 .
$$

Note that the eigenvalues of $H$ are just shifted by $\varepsilon^{-2} E_{1}$ with respect to those of $H_{\text {ren }}$, while the eigenfunctions coincide.

Let us remark at this point that, based on our estimates, it is possible to give explicit threshold values for the smallness of $\varepsilon$ (in terms of the geometry of $\Sigma$, the magnetic field, and the isolation distance of $\mu$ ). However, we have preferred this more concise presentation for the sake of readability of the paper.

\section{Acknowledgment}

Support by the Institute Mittag-Leffler (Djursholm, Sweden), where this paper was being prepared, is gratefully acknowledged. The work has been partially supported by the projects RVO61389005 and RVO68407700; and the grants No. P203/11/0701 and No. 13-11058S of the Czech Science Foundation (GAČR). 


\section{References}

[1] G. Carron, P. Exner, and D. Krejčiřík, Topologically nontrivial quantum layers, J. Math. Phys. 45 (2004), 774-784.

[2] R. C. T. da Costa, Quantum mechanics of a constrained particle, Phys. Rev. A 23 (1981), 1982-1987.

[3] _ Constraints in quantum mechanics, Phys. Rev. A 25 (1982), 2893-2900.

[4] P. Duclos and P. Exner, Curvature-induced bound states in quantum waveguides in two and three dimensions, Rev. Math. Phys. 7 (1995), 73-102.

[5] P. Duclos, P. Exner, and D. Krejčiřík, Bound states in curved quantum layers, Comm. Math. Phys. 223 (2001), 13-28.

[6] P. Duclos, P. Št'ovíček, and M. Tušek, On the two-dimensional Coulomb-like potential with a central point interaction, J. Phys. A 43 (2010).

[7] T. Ekholm and H. Kovařík, Stability of the magnetic Schrödinger operator in a waveguide, Comm. in Partial Differential Equations 30 (2005), no. 4, 539-565.

[8] P. Exner and D. Krejčiřík, Bound states in mildly curved layers, J. Phys. A $\mathbf{3 4}$ (2001), 5969-5985.

[9] P. Exner and P. Šeba, Bound states in curved quantum waveguides, J. Math. Phys. 30 (1989), 2574-2580.

[10] P. Freitas and D. Krejčiřík, Location of the nodal set for thin curved tubes, Indiana Univ. Math. J. 57 (2008), no. 1, 343-376.

[11] R. Froese and I. Herbst, Realizing holonomic constraints in classical and quantum mechanics, Comm. Math. Phys. 220 (2001), 489-535.

[12] N. E. Hurt, Mathematical physics of quantum wires and devices, Kluwer, Dordrecht, 2000.

[13] H. Jensen and H. Koppe, Quantum mechanics with constraints, Ann. Phys. 63 (1971), 586-591.

[14] T. Kato, Perturbation theory for linear operators, Springer-Verlag, Berlin, 1966.

[15] D. Krejčiř́ík and J. Křǐž, On the spectrum of curved quantum waveguides, Publ. RIMS, Kyoto University 41 (2005), no. 3, 757-791.

[16] D. Krejčiřík and H. Šediváková, The effective Hamiltonian in curved quantum waveguides under mild regularity assumptions, Rev. Math. Phys. 24 (2012), 1250018

[17] W. Kühnel, Differential geometry, AMS, Providence, Rhode Island, 2006.

[18] J. Lampart, S. Teufel, and J. Wachsmuth, Effective Hamiltonians for thin Dirichlet tubes with varying cross-section, Mathematical Results in Quantum Physics, Hradec Králové, 2010, xi+274 p., World Scientific, Singapore, 2011, pp. 183-189.

[19] A. Laptev and T. Weidl, Hardy inequalities for magnetic Dirichlet forms, Oper. Theory Adv. Appl. 108 (1999), 299-305.

[20] E. H. Lieb and M. Loss, Analysis, American Mathematical Society, Providence, Rhode Island, 1997.

[21] Ch. Lin and Z. Lu, On the discrete spectrum of generalized quantum tubes, Comm. Partial Differential Equations 31 (2006), 1529-1546.

$[22] \_$Existence of bound states for layers built over hypersurfaces in $\mathbb{R}^{n+1}$, J. Funct. Anal. 244 (2007), 1-25.

[23] _ Quantum layers over surfaces ruled outside a compact set, J. Math. Phys. 48 (2007), Art. No. 053522. 
[24] J. T. Londergan, J. P. Carini, and D. P. Murdock, Binding and scattering in two-dimensional systems, LNP, vol. m60, Springer, Berlin, 1999.

[25] Z. Lu and J. Rowlett, On the discrete spectrum of quantum layers, J. Math. Phys. 53 (2012), 073519.

[26] K. A. Mitchell, Gauge fields and extrapotentials in constrained quantum systems, Phys. Rev. A 63 (2001), art. 042112.

[27] M. Nakahara, Geometry, topology, and physics, Taylor \& Francis Group, London, 2003.

[28] M. Reed and B. Simon, Methods of Modern Mathematical Physics, Vol. I, Academic Press, London, 1980.

[29] B. Simon, Quantum mechanics for Hamiltonians defined by quadratic forms, Princeton Univ. Press., New Jersey, 1971.

[30] M. Spivak, A comprehensive introduction to differential geometry, vol. II, Publish or Perish, Houston, Texas, 1999.

[31] _ A comprehensive introduction to differential geometry, vol. I, Publish or Perish, Houston, Texas, 2005.

[32] G. Teschl, Mathematical methods in quantum mechanics, AMS, Providence, Rhode Island, 2009.

[33] J. Tolar, On a quantum mechanical d'Alembert principle, Group theoretical methods in physics, LNP, vol. 313, Springer, 1988, pp. 268-274.

[34] J. Wachsmuth and S. Teufel, Effective Hamiltonians for constrained quantum systems, preprint on arXiv:0907.0351v3 [math-ph] (2009).

[35] _ Constrained quantum systems as an adiabatic problem, Phys. Rev. A 82 (2010), 022112.

[36] O. Wittich, L2-homogenization of heat equations on tubular neighborhoods, arXiv:0810.5047 [math.AP] (2008). 